\title{
Land Surface Temperature and Emissivity Separation from Cross-Track Infrared Sounder Data with Atmospheric Reanalysis Data and ISSTES Algorithm
}

\author{
Yu-Ze Zhang, ${ }^{1}$ Xiao-Guang Jiang, ${ }^{1,2,3}$ Hua Wu, ${ }^{1,2,4}$ Ya-Zhen Jiang, \\ Zhao-Xia Liu, ${ }^{5}$ and Cheng Huang ${ }^{1}$ \\ ${ }^{1}$ University of Chinese Academy of Sciences, Beijing 100049, China \\ ${ }^{2}$ State Key Laboratory of Resources and Environment Information System, \\ Institute of Geographic Sciences and Natural Resources Research, Chinese Academy of Sciences, Beijing 100101, China \\ ${ }^{3}$ Key Laboratory of Quantitative Remote Sensing Information Technology, Academy of Opto-Electronics, Chinese Academy of Sciences, \\ Beijing 100094, China \\ ${ }^{4}$ Jiangsu Center for Collaborative Innovation in Geographical Information Resource Development and Application, \\ Nanjing 210023, China \\ ${ }^{5}$ Xinjiang Institute of Ecology and Geography, Chinese Academy of Sciences, Wulumuqi, Xinjiang 830011, China
}

Correspondence should be addressed to Hua Wu; wuhua@igsnrr.ac.cn

Received 9 March 2017; Revised 7 June 2017; Accepted 21 June 2017; Published 21 August 2017

Academic Editor: Anthony R. Lupo

Copyright (c) $2017 \mathrm{Yu}-\mathrm{Ze}$ Zhang et al. This is an open access article distributed under the Creative Commons Attribution License, which permits unrestricted use, distribution, and reproduction in any medium, provided the original work is properly cited.

\begin{abstract}
The Cross-track Infrared Sounder (CrIS) is one of the most advanced hyperspectral instruments and has been used for various atmospheric applications such as atmospheric retrievals and weather forecast modeling. However, because of the specific design purpose of CrIS, little attention has been paid to retrieving land surface parameters from CrIS data. To take full advantage of the rich spectral information in CrIS data to improve the land surface retrievals, particularly the acquisition of a continuous Land Surface Emissivity (LSE) spectrum, this paper attempts to simultaneously retrieve a continuous LSE spectrum and the Land Surface Temperature (LST) from CrIS data with the atmospheric reanalysis data and the Iterative Spectrally Smooth Temperature and Emissivity Separation (ISSTES) algorithm. The results show that the accuracy of the retrieved LSEs and LST is comparable with the current land products. The overall differences of the LST and LSE retrievals are approximately $1.3 \mathrm{~K}$ and $1.48 \%$, respectively. However, the LSEs in our study can be provided as a continuum spectrum instead of the single-channel values in traditional products. The retrieved LST and LSEs now can be better used to further analyze the surface properties or improve the retrieval of atmospheric parameters.
\end{abstract}

\section{Introduction}

Land Surface Temperature and Emissivity (LST and LSE) are two key parameters in quantitative remote sensing and have been widely used in many fields such as meteorological and climate models, lithological mapping, and resources exploration $[1,2]$.

Over recent decades, great effort has been made to retrieve the LST and LSEs from multispectral thermal infrared (TIR) data, and some typical algorithms have been successfully used to determine the LST and LSEs from space measurements [3]. For example, the Split Window algorithm and Day/Night algorithm are used for the Moderate Resolution Imaging Spectroradiometer (MODIS), and the Temperature and Emissivity Separation (TES) algorithm is used for the Advanced Spaceborne Thermal Emission and Reflection Radiometer (ASTER) [4-7]. According to the current multispectral land products, the general accuracy of the LST can be approximately $1 \mathrm{~K}$ [7-10]. However, in most algorithms, some empirical constraints on LSE are also 
needed to determine the LST, such as prior knowledge of the LSE in the Split Window (SW) algorithm and the assumption of a constant LSE during night and day in the Day/Night $(\mathrm{D} / \mathrm{N})$ algorithm $[4,5]$. Unlike the LST product, the current LSE products are usually determined according to a classification map, which makes the accuracy of the LSE product highly dependent on prior knowledge $[8,10]$. In addition, due to the characteristics of multispectral instruments, only a few broadband LSEs can be provided for the users [11].

Compared with the multispectral instruments, the hyperspectral instruments are usually designed with hundreds and thousands of channels and provide a new direction to retrieve LST and LSEs by solving the ill-posed Radiative Transfer Equation (RTE) [12-14]. The typical algorithms are the Iterative Spectrally Smooth Temperature and Emissivity Separation (ISSTES) algorithm and the Linear Emissivity Constraint Temperature and Emissivity Separation (LECTES) algorithm $[12,13]$. Unlike the multispectral algorithms, due to the characteristics of hyperspectral data, a continuous LSE spectrum can be theoretically obtained with the hyperspectral algorithms, which is critical for further analyzing the land surface and atmospheric properties.

Currently, the hyperspectral data can be obtained from several sensors: the Atmospheric Infrared Sounder (AIRS) on Earth-observing system satellites [15], the Interferometer Atmospheric Sounding Instrument (IASI) on the European meteorological operational satellite program-A (METOPA) $[16,17]$, and the Cross-track Infrared Sounder (CrIS) on the next-generation National Polar-orbiting Operational Environmental Satellite System [18]. Among them, the CrIS, which was launched in 2011, is the most advanced and is also regarded as the next series of the AIRS. However, similar to the other hyperspectral instruments, the CrIS was originally designed for atmospheric applications. In other words, the CrIS will generally produce high-resolution, threedimensional temperature, pressure, and moisture profiles, and little information about the land surface is available for the users.

Actually, in current CrIS atmospheric retrievals, both the land surface parameters and the atmospheric profiles are simultaneously acquired for each Field-of-Regard (FOR) through a maximum-likelihood method [19]. However, due to the specific design purpose of CrIS, only the pressure, temperature, and moisture profiles are taken as the required outputs, while the land surface parameters, which are not currently produced, are regarded as optional outputs [20]. In addition, it is also reported that, even for the retrieved LSEs in current CrIS simultaneous retrievals, there are still only a few channel values that can be acquired, and thus the results are still similar to the multispectral products. Therefore, to take full advantage of the rich spectral information in CrIS data to improve the land surface retrievals, particularly the acquisition of a continuous LSE spectrum, this paper makes an effort to simultaneously retrieve a continuous LSE spectrum and the LST from the CrIS data with the atmospheric reanalysis data and the ISSTES algorithm. The following sections are organized as follows. In Section 2, the study area and data used in this paper are presented. In Section 3, the method for atmospheric correction and
TABLE 1: Spectral resolution, frequency range, and number of channels of CrIS SDRs.

\begin{tabular}{lccc}
\hline Band & $\begin{array}{c}\text { Resolution } \\
\left(\mathrm{cm}^{-1}\right)\end{array}$ & $\begin{array}{c}\text { Frequency } \\
\text { range }\left(\mathrm{cm}^{-1}\right)\end{array}$ & $\begin{array}{c}\text { Number of } \\
\text { channels }\end{array}$ \\
\hline LWIR & 0.625 & $650-1095$ & 713 \\
MWIR & 1.25 & $1210-1750$ & 433 \\
SWIR & 2.5 & $2155-2550$ & 159 \\
\hline
\end{tabular}

the method for Temperature and Emissivity Separation are briefly described. Section 4 gives the results and Section 5 gives the discussion. Conclusions are provided in Section 6.

\section{Study Area and Data}

2.1. Study Area. To find a mostly cloud-free region, a study area with longitude from $1.4^{\circ} \mathrm{W}$ to $7.9^{\circ} \mathrm{W}$ and latitude from $38^{\circ} \mathrm{N}$ to $43^{\circ} \mathrm{N}$ is selected. The map of the study area generated from the Global Land Cover 2000 map produced by the Institute of Environment and Sustainability (IES) is shown in the red rectangle in Figure 1 [21]. In this area, the main land covers are cultivated and managed areas, tree cover, shrub cover, artificial surfaces, and associated areas.

\subsection{Cross-Track Infrared Sounder Sensor Data Records (CrIS} SDRs). The CrIS is a Fourier Transform Spectrometer (FTS) onboard the Suomi National Polar-Orbiting Partnership (SNPP) Satellite, which was launched on October 28, 2011. It is designed with 1305 spectral channels over three wavelength ranges (see Table 1) and has a spatial resolution of $14 \mathrm{~km}$ at nadir.

Two data formats are currently provided by CrIS: the interferogram measurements and calibration data in the form of Raw Data Records (RDRs) and the calibrated and geolocated data in the form of Sensor Data Records (SDRs). In this paper, only the LWIR band in CrIS SDRs, which were acquired in 2013, is actually used to retrieve the LST and LSEs. All the data can be obtained from NOAA's Comprehensive Large Array-data Stewardship System (CLASS) [22, 23].

\subsection{Data for Atmospheric Correction: Global Data Assimila-} tion System (GDAS). The Global Data Assimilation System (GDAS) is used by National Center for Environmental Prediction (NCEP) to place observations into a gridded model space to start or initialize weather forecasts with observed data. Dozens of atmospheric and land-soil variables are available in this dataset, from temperature, winds, and precipitation to soil moisture and atmospheric ozone concentration. In this paper, the GDAS analysis data in GRIB2 format is used, which is on a $1^{\circ} \times 1^{\circ}$ longitude/latitude grid and generated globally every $6 \mathrm{~h}$ (0:00, 06:00, 12:00, and 18:00 UTC) [24].

\subsection{Data for Cross-Validation}

2.4.1. Visible Infrared Imaging Radiometer Suite Environment Data Records (VIIRS EDRs). The Visible Infrared Imaging 


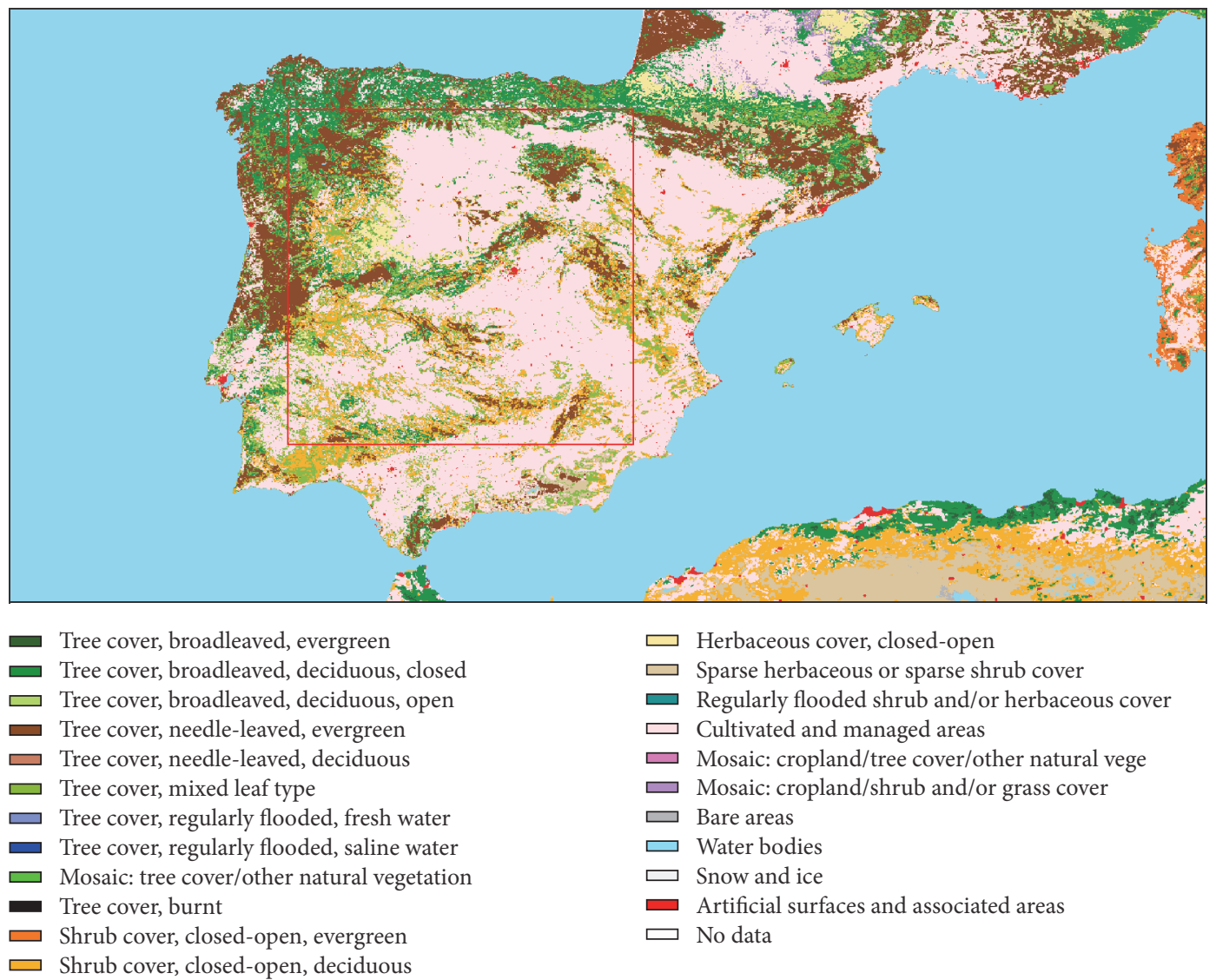

FIGURE 1: Land surface covers in study area with a resolution of 1 kilometer generated from the Global Land Cover 2000 map.

Radiometer Suite (VIIRS) is one of the five major Earthobserving instruments onboard the S-NPP. It provides full daily coverage on both the day and night sides of the Earth. VIIRS has 22 spectral bands covering the spectrum between $0.412 \mu \mathrm{m}$ and $12.01 \mu \mathrm{m}$, including 16 moderate resolution bands (M-bands) with a spatial resolution of $750 \mathrm{~m}$ at nadir, 5 imaging resolution bands (I-bands) with a spatial resolution of $375 \mathrm{~m}$ at nadir, and one panchromatic day/night band (DNB) with a $750 \mathrm{~m}$ spatial resolution throughout the sea [25].

In this paper, the VIIRS LST EDRs are used for validating the LST retrievals, which are calculated with two types of algorithms: split-windows (SW) and optional dual-split window (DSW) [10]. Compared with the ground measurements in four sites (Gobi, sand dune, and desert steppe), the average root mean square error (RMSE) and bias of VIIRS LSTs were approximately $2.74 \mathrm{~K}$ and $0.36 \mathrm{~K}$ in daytime and approximately $1.48 \mathrm{~K}$ and $-0.58 \mathrm{~K}$ in nighttime, respectively [26]. Although, as reported in [27], two problems were identified in the VIIRS LST EDR: (1) inaccurate spectral emissivity values used in the VIIRS algorithm, which are derived from fixed values depending on a limited number of surface types; (2) a nonrepresentative algorithm coefficients under hot and very humid conditions, the data used in our study are still reliable because most of the surfaces in our study area were the typical ones and the water vapor contents of the corresponding atmospheric profiles were all less than $3 \mathrm{~g} / \mathrm{cm}^{2}$.

\subsubsection{Atmospheric Infrared Sounder (AIRS) Emissivity Prod-} uct. Unlike the validation for LSTs, because no LSE product can be provided by the instruments onboard the S-NPP, the AIRS, which was also used as a reference instrument in designing the CrIS, is selected in our study for evaluating the LSE retrievals.

The AIRS is one of six instruments aboard Aqua, which was launched into the Earth orbit on May 4, 2002. It consists of 2378 infrared channels that cover 3.7-15.4 $\mu \mathrm{m}$ with a spatial resolution of $13.5 \mathrm{~km}$ at nadir [15].

In our study, only the Version 6 (v6) AIRS standard surface product, which contains retrieved estimates of the surface properties with a spatial resolution of $50 \mathrm{~km}$ and cloud and atmospheric information [28], is used for validating the LSEs. The current v6 surface retrieval algorithm actually uses a regression plus simultaneous solution approach to retrieve the spectral LSE, bidirectional reflectance, and LST. It was reported that, over Namib Desert, the absolute LSE differences between AIRS LSE product and ASTER and Lab data were 2.32 and $2.26 \%$, respectively. Over Kalahari Desert, the differences were 0.68 and $2.26 \%$ [29]. 


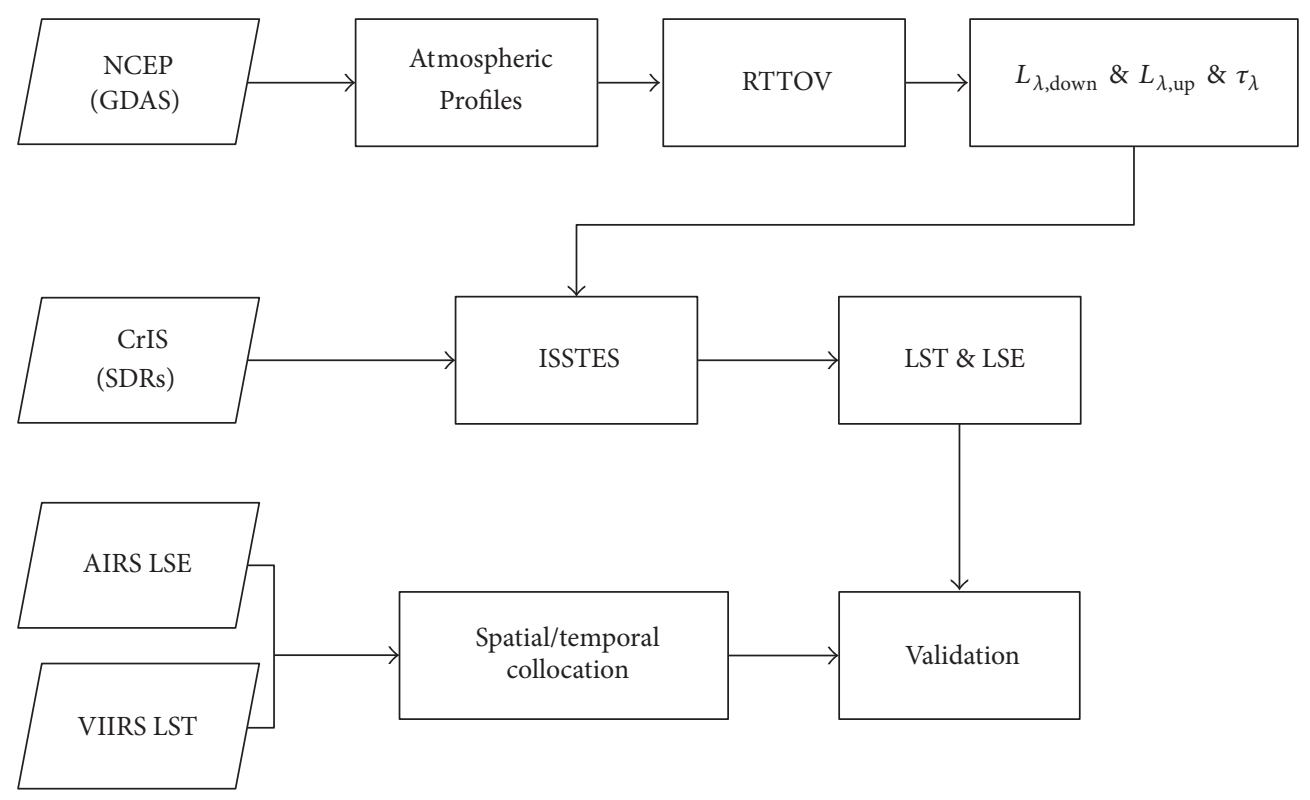

FIGURE 2: Flowchart of Temperature and Emissivity Separation with CrIS data.

\section{Methodology}

3.1. Basic Theory and Scheme. On the basis of radiative transfer theory, under clear-sky conditions the spectral radiance measured in the thermal infrared (TIR) band at the sensor level can be written as

$$
\begin{aligned}
L_{i, \text { at-sensor }}= & \varepsilon_{i} \cdot B_{i}\left(T_{s}\right) \cdot \tau_{i}+\left(1-\varepsilon_{i}\right) \cdot L_{i, \text { down }} \cdot \tau_{i} \\
& +L_{i, \text { up }}
\end{aligned}
$$

where $i$ is the TIR channel; $L_{i \text {,at-sensor }}$ is the radiance at the sensor level; $\varepsilon_{i}$ is the Land Surface Emissivity; $T_{s}$ is the Land Surface Temperature; $B_{i}\left(T_{s}\right)$ is the Planck Function at the temperature $T_{s} ; L_{i \text {,down }}$ and $L_{i \text {,up }}$ are the downwelling and upwelling atmospheric radiance, respectively; $\tau_{i}$ is the total atmospheric transmittance.

It is clear that if one wants to know the LST and LSEs, the atmospheric parameters in (1) must be provided in advance, which is also known as the process of atmospheric correction. For this purpose, the GDAS atmospheric reanalysis products and the fast radiative transfer model RTTOV are used in this paper [30].

Then, one can easily find that there are still $N+1$ unknowns ( $N$ LSEs and 1 LST for $N$ channels) in the RTE with only $N$ equations; therefore, a Temperature and Emissivity Separation (TES) solution, such as the ISSTES algorithm in this paper, is also necessary for determining the LST and LSEs [31-33].

For the validations in our study, because no appropriate ground measurements at large scales for the study area are available, the cross-validation is used with the VIIRS LST EDRs and the AIRS emissivity product. In addition, due to different spatial resolutions between the VIIRS $(750 \mathrm{~m}$ at nadir) and CrIS (14 km at nadir) data, a spatial collocation also needs to be done before the cross-validation. The same process is included in the cross-validation with the AIRS product too, which has a spatial resolution of $50 \mathrm{~km}$ at nadir.

The overall flowchart of this paper is shown in Figure 2.

3.2. Atmospheric Correction. First, an interpolated NCEP profile from the global dataset should be obtained according to the coordinate and acquisition time of the CrIS pixel. Next, obtain the entire pressure, temperature, and moisture profiles right above the ground and then run the RTTOV, a fast radiative transfer model, to calculate the atmospheric radiance values $\left(L_{i, \text { down }}\right.$ and $\left.L_{i \text {,up }}\right)$ and transmittances $\left(\tau_{i}\right)$. Here, to ensure the final accuracy of TES, the TIR band used in this paper is $800-970 \mathrm{~cm}^{-1}$, which excludes the region with obvious ozone absorption.

3.3. Temperature and Emissivity Separation (ISSTES). After correcting the atmosphere, the land leaving radiance can be calculated by

$$
\begin{aligned}
L_{i, \text { land_leaving }} & =\frac{L_{i \text {,at_sensor }}-L_{i, \text { up }}}{\tau_{i}} \\
& =\varepsilon_{i} \cdot B_{i}\left(T_{s}\right)+\left(1-\varepsilon_{i}\right) \cdot L_{i, \text { down }},
\end{aligned}
$$

where $L_{i \text {,land_leaving }}$ is the land leaving radiance at the surface level.

Now, only $\varepsilon_{i}$ and $T_{s}$ are unknown in the RTE and $\varepsilon_{i}$ can be rewritten as a function of $T_{s}$ :

$$
\varepsilon_{i}=\frac{L_{i, \text { land_leaving }}-L_{i, \text { down }}}{B_{i}\left(T_{s}\right)-L_{i, \text { down }}} .
$$

According to (3), if $T_{s}$ is not accurately estimated, the corresponding LSE spectrum will exhibit sharp convexities or concavities, which are called atmospheric features that are 
caused by gas absorption [33]. Because a typical emissivity spectrum is smoother than that with atmospheric features, the ISSTES algorithm has been used to iteratively retrieve the LSEs and LST from hyperspectral TIR data [12].

Firstly, an initial estimate $T_{0}$ should be provided to calculate the initial LSEs, such as the max value of surface brightness temperatures. Next, a cost function should be defined for determining the LST and LSEs:

$$
F=\sum_{i=1}^{N}\left(L_{i, \text { land_leaving }}^{\prime}-L_{i, \text { land_leaving }}\right)^{2},
$$

with

$$
\begin{aligned}
L_{i, \text { land_leaving }}^{\prime} & =\varepsilon_{i, S M} \cdot B_{i}\left(T_{0}\right)+\left(1-\varepsilon_{i, S M}\right) \cdot L_{i, \text { down }}, \\
\varepsilon_{i, S M} & =\frac{\varepsilon_{i-1}+\varepsilon_{i}+\varepsilon_{i+1}}{3},
\end{aligned}
$$

where $\varepsilon_{i-1}, \varepsilon_{i}$, and $\varepsilon_{i+1}$ are three neighbor channels in the TIR band; $F$ is the cost function; $L_{i \text {,land_leaving }}$ is the measured radiance from CrIS; $L_{i, \text { land_leaving }}^{\prime}$ is the estimated radiance at the sensor level with the emissivity of $\varepsilon_{i, S M}$ in each iteration.

Then, the optimal estimate of LST can be obtained by searching the minimum of the cost function $F$ through an iterative process by changing $T_{0}$. After we obtain this optimal LST, the LSE spectrum can be easily derived from (3). Although some different cost functions for determining the optimal LST and LSEs have been proposed, it is found that the performance of ISSTES is not sensitive to the choice of the cost function $[30,31]$.

Beyond that, a constraint of the maximum and minimum value of emissivity between 0 and 1 is also used in our study to ensure that all retrieved LSEs are in the physical range.

\section{Results and Analysis}

4.1. Cross-Validation for Land Surface Temperature (LST). For the LST validation, as both VIIRS and CrIS are onboard the S-NPP, it is not necessary to consider the errors caused by the acquisition time. However, the different spatial resolution between CrIS and VIIRS data may lead to a scale effect problem $[34,35]$. A simple collocation in space still needs to be performed in advance.

Firstly, for a given CrIS pixel, all the VIIRS pixels that fall in the spatial range of the CrIS pixel are selected. Next, all of the pixels with high quality (HQ) are selected through the VIIRS quality flag (QF). Finally, an arithmetic mean of the VIIRS LSTs is used as the actual CrIS LST for the given pixel. It should be noted that the calculated mean LST might be not representative if there are no sufficient valid VIIRS pixels with high quality. To solve this problem, a percent of HQ pixels (90\%) is set to be a constraint to guarantee the credibility of the calculation of real LST. Furthermore, because of the lower spatial heterogeneity at nighttime, all datasets in this paper are acquired at nighttime to decrease the effect of the space matching $[26,36]$. In addition, to better evaluate the results under different atmospheric conditions, 4 different months were selected to retrieve the LST and LSE, which also indicate the seasons of a year.
Figure 3 shows the comparison of the retrieved LSTs and VIIRS LSTs, and totally 29 days from 4 different months in 2013 were selected.

For each month, the Root Mean Square Deviation (RMSD) of LSTs is approximately $0.84 \mathrm{~K}$ in February, and the bias is $-0.07 \mathrm{~K}$. In May, the RMSD and BIAS are approximately $1.39 \mathrm{~K}$ and $0.17 \mathrm{~K}$, respectively. An RMSD of $1.63 \mathrm{~K}$ and a BIAS of $-0.15 \mathrm{~K}$ are obtained in August. For November, the RMSD and BIAS are approximately $1.15 \mathrm{~K}$ and $-0.11 \mathrm{~K}$, respectively. The overall RMSD of the LST retrievals in August is the worst in the 4 selected months, which might be caused by the higher water vapor content (WVC) in August. However, according to the results, the overall RMSD of the 4 month is only approximately $1.34 \mathrm{~K}$ with a bias of $-0.06 \mathrm{~K}$. In addition, the results in Figure 3 also show an obvious seasonal change on LSTs in a year.

4.2. Cross-Validation for the Land Surface Emissivity (LSE). For the LSE validation, due to the different characteristics of AIRS and CrIS, such as the different spatial and temporal resolutions, there are not as many points as LSTs for crossvalidation. In fact, only the AIRS pixels with sufficient CrIS pixels in them, which cover $85 \%$ of the AIRS pixel range, are used for the cross-validation. In addition, a time constraint of 6 hours for acquisition time is also included in our study, but no limitations on the view angle are taken into account for obtaining a basic number of valid pixels.

To directly compare CrIS LSEs with AIRS, we interpolated the CrIS LSEs into identical channels in the AIRS LSE product from 800 to $970 \mathrm{~cm}^{-1}: 819.67 \mathrm{~cm}^{-1}$ (B1), $847.46 \mathrm{~cm}^{-1}$ (B2), $877.19 \mathrm{~cm}^{-1}$ (B3), $909.09 \mathrm{~cm}^{-1}$ (B4), and $943.4 \mathrm{~cm}^{-1}$ (B5). Figure 4(a) shows the comparison of the retrieved LSEs and AIRS product at each band, and Figure 4(b) shows a histogram of the differences between retrieved LSEs and AIRS LSEs. Detailed information of the comparison is provided in Table 2.

According to Table 2, the total RMSDs of the LSEs at each band are approximately $1.22-1.66 \%$. The RMSD obviously decreases with the decrease (increase) in wavenumber (wavelength). The reason may be that at small wavenumbers the atmosphere is commonly much more transparent than at large wavenumbers in the selected TIR band.

From Figure 4(a), it is clear that the LSEs retrieved from November were with an obvious bias error, which indicates the ISSTES algorithm was more sensitive to the atmospheric correction under dry-cold conditions. Besides that, some singular points, which are circled with red lines, are also easily found in Figure 4(a), and all of them are from the month of May. For these points, the maximum difference of the channel LSEs can be larger than 5\%, and there are many possible reasons for this: (1) the poor quality of the retrieved LSTs of the selected pixels; (2) the obvious differences in view angle between the CrIS and AIRS pixels; (3) the singular points caused by the inversion of RTE when the LST is close to the equivalent atmospheric temperature. However, the statistical results show that the total percentage of LSEs at each band remains larger than $80 \%$ with a mean difference of less than $2 \%$. Details are shown in Table 3. 


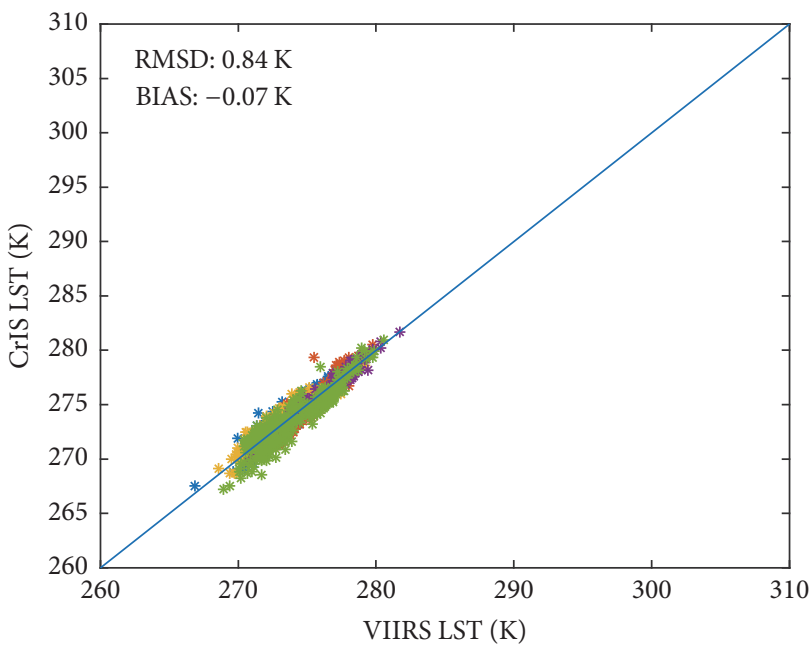

(a)

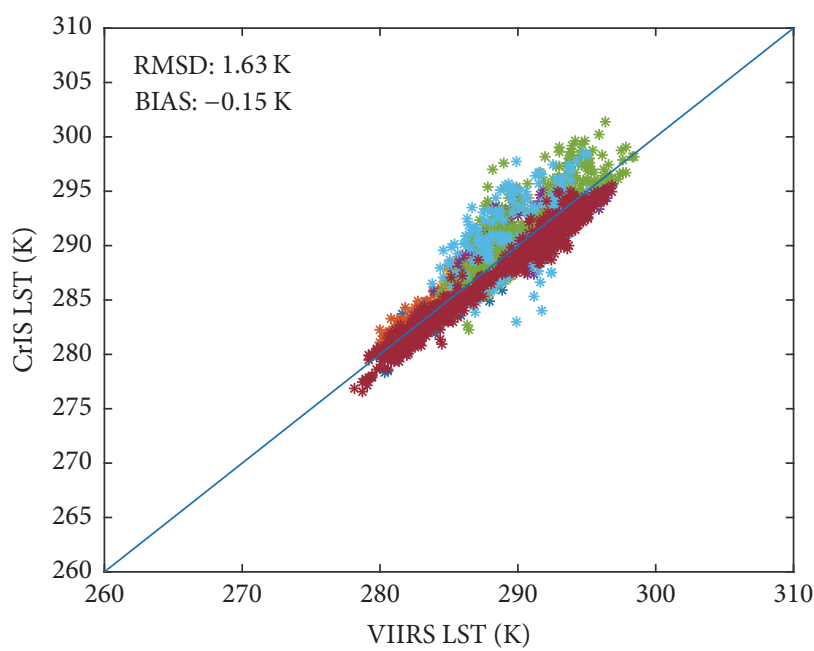

(c)

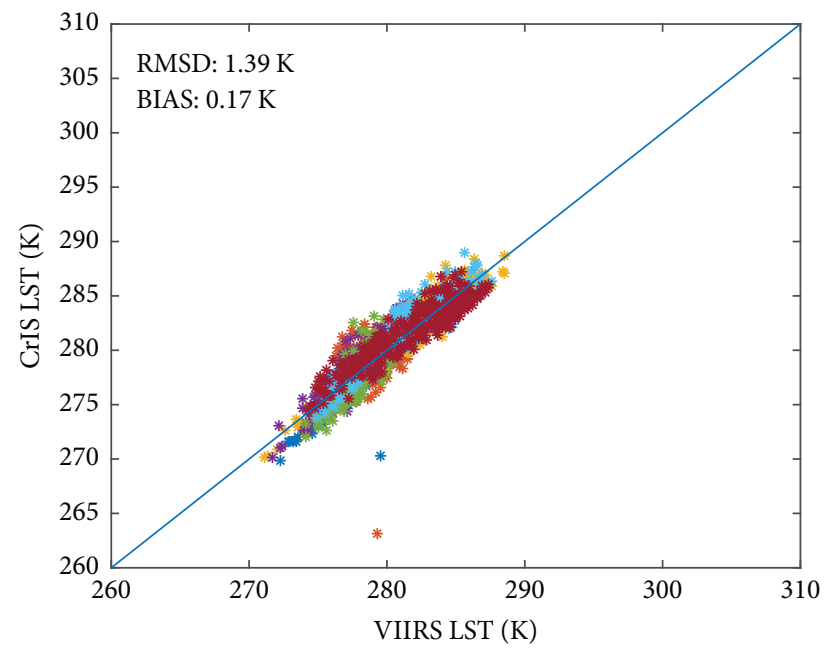

(b)

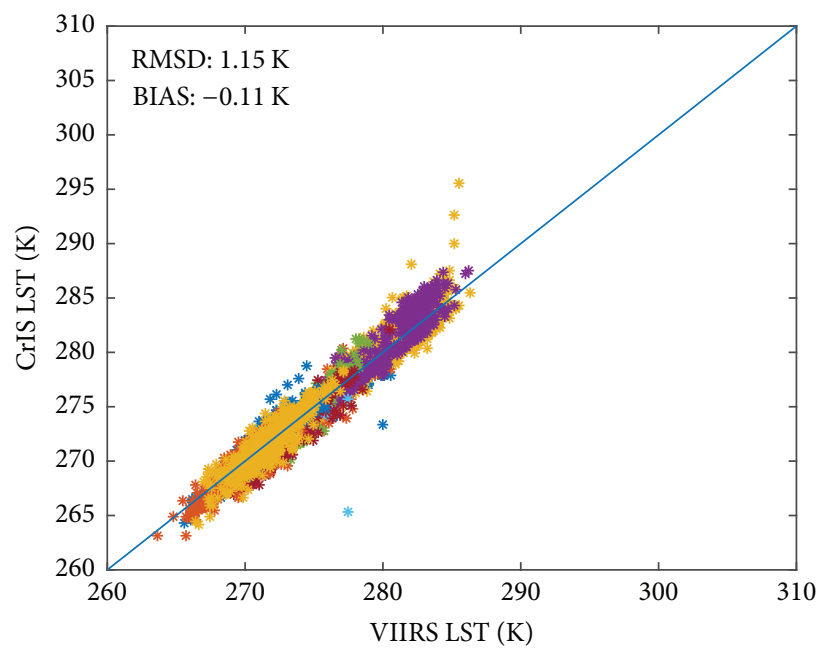

(d)

FIGURE 3: Results of retrieved LSTs from CrIS data in 2 different months, 2013. Different colors indicate the different days: (a) LSTs in February; (b) LSTs in May; (c) LSTs in August; (d) LSTs in November.

TABLE 2: Differences between CrIS and AIRS LSE product at 5 AIRS TIR bands.

\begin{tabular}{|c|c|c|c|c|c|c|c|c|c|c|}
\hline \multirow{2}{*}{ Band $\left(\mathrm{cm}^{-1}\right)$} & \multicolumn{2}{|c|}{819.67} & \multicolumn{2}{|c|}{847.46} & \multicolumn{2}{|c|}{877.19} & \multicolumn{2}{|c|}{909.09} & \multicolumn{2}{|c|}{943.4} \\
\hline & RMSE & BIAS & RMSE & BIAS & RMSE & BIAS & RMSE & BIAS & RMSE & BIAS \\
\hline May (\%) & 1.66 & -0.03 & 1.70 & 0.00 & 1.87 & -0.29 & 2.12 & -0.80 & 2.00 & -0.39 \\
\hline Nov. (\%) & 1.11 & 0.81 & 1.39 & 1.16 & 1.44 & 1.21 & 1.41 & 1.16 & 1.60 & 1.37 \\
\hline Total (\%) & 1.22 & 0.62 & 1.42 & 0.93 & 1.48 & 0.94 & 1.57 & 1.05 & 1.66 & 1.10 \\
\hline
\end{tabular}

TABLE 3: The detailed statistical information of LSEs at each of the 5 bands.

\begin{tabular}{llllll}
\hline$\Delta$ LSE & Band 1 & Band 2 & Band 3 & Band 4 & Band 5 \\
\hline$\leq 2 \%$ & $95.97 \%$ & $89.26 \%$ & $87.25 \%$ & $85.23 \%$ & $83.22 \%$ \\
$\leq 1.5 \%$ & $86.58 \%$ & $73.83 \%$ & $70.47 \%$ & $63.76 \%$ & $38.39 \%$ \\
$\leq 1 \%$ & $65.10 \%$ & $42.28 \%$ & $44.30 \%$ & $36.24 \%$ & $31.54 \%$ \\
\hline
\end{tabular}




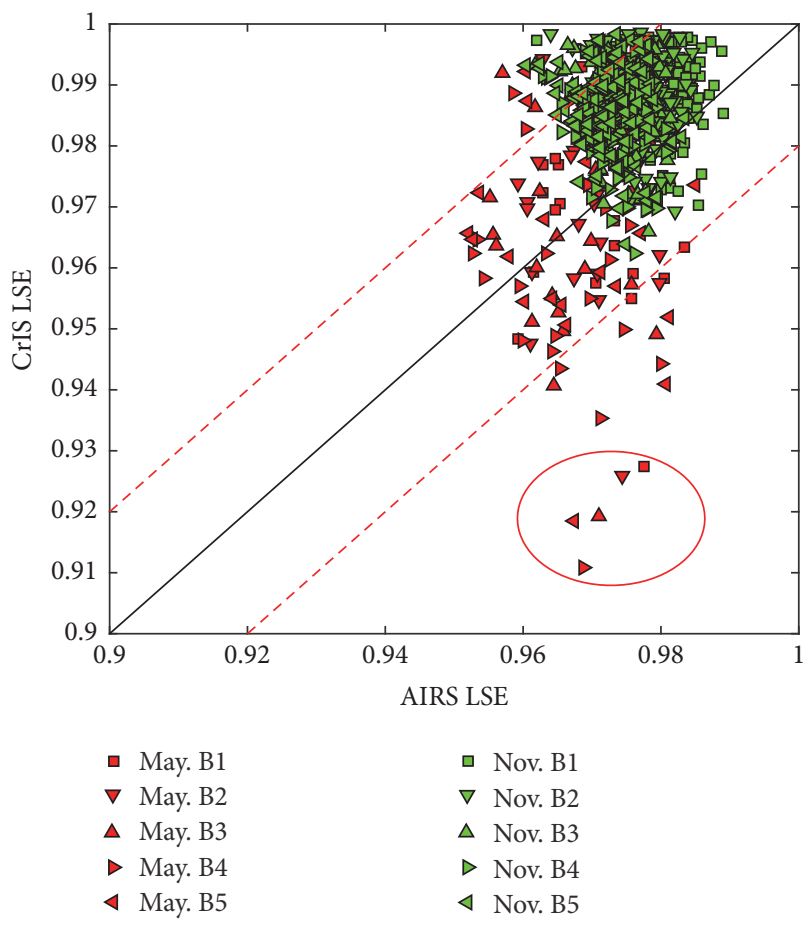

(a)

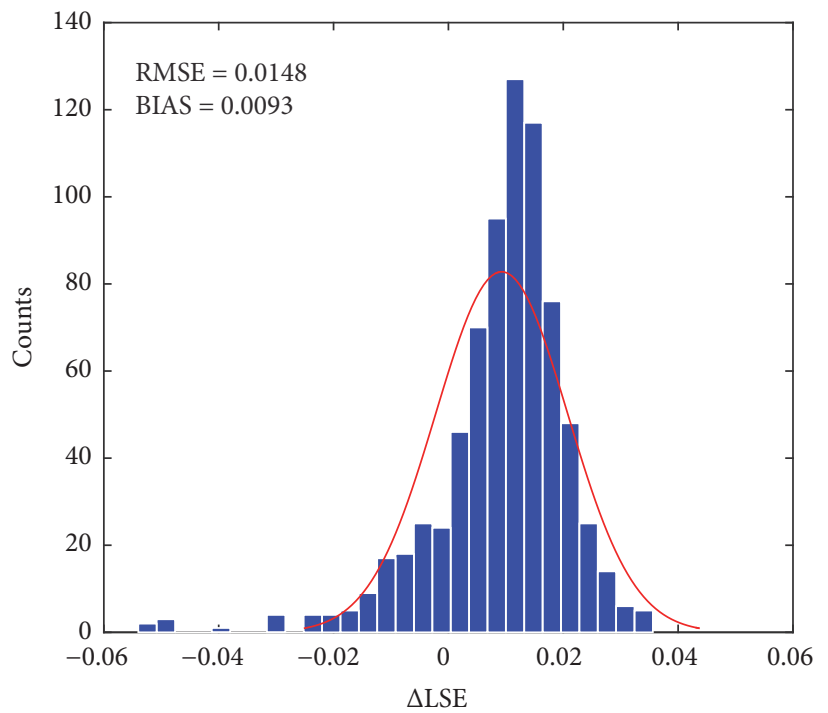

(b)

FIgURE 4: The comparisons of LSEs between CrIS and AIRS: (a) scatterplots of LSEs in five bands for 2 months; (b) histograms of LSE differences between CrIS and AIRS. The red dashed lines mean the points that have a LSE difference of 0.02; the points circled with red solid lines are the singular ones.

In general, the overall accuracy of the LSE retrievals is comparable with the AIRS LSE product. According to the results in [27], the maximum error of AIRS product over desert was approximately $2 \%-3 \%$. That is, for soil surfaces, the maximum error of the retrieved LSEs from CrIS data is approximately $2.3 \%-3.4 \%$. This specific value will be even lower for other surfaces such as vegetation. On the other hand, unlike the few channel LSE values provided in the AIRS product, a continuum LSE spectrum is obtained simultaneously with the LST in our study. To show this result, a comparison of the mean LSEs of the selected pixels in May is shown in Figure 5. The solid red line indicates the mean ARIS LSEs, and the dotted red lines show the standard deviation of the mean LSEs. Similarly, the solid and dotted blue lines are the mean and standard deviation of the original CrIS LSEs, respectively. However, due to the misfits on atmospheric profiles, the LSE spectrum may be fluctuated by using the ISSTES algorithm. To decrease the uncertainty of atmospheric profiles on atmospheric correction and make the retrieved LSEs more practical for applications, a smoothed LSE spectrum, which is also showed in Figure 5 with green line, can be then obtained by using some mathematic methods.

As shown, when the mean LSEs are compared, the retrieved LSEs exhibit good shape consistency with the AIRS product. Different from the 5 channel LSEs in AIRS product in the range of $800-970 \mathrm{~cm}^{-1}$, more spectral features can be contained in the retrieved LSE spectrum. It is clear that the
AIRS LSE spectrum is more close to a near-gray surface, while obvious spectral changes, which are valuable for further analyses of atmospheric and surface properties, can be easily found in our retrieved LSE spectrum.

\section{Discussion}

Currently, a simultaneous solution approach is used to retrieve the atmospheric profiles together with the LST and LSEs from the CrIS data [20]. To better eliminate the influence of the cloud, a Cloud-Clearing (CC) technique, which utilizes the CrIS radiance values within a FOR $(3 \times$ 3 Filed-Of-View, FOV), is included in the retrieval process. Thus, the released CrIS products are produced with a lower spatial resolution approximately $45 \mathrm{~km}$. Due to the original purpose of the CrIS, the present approach focuses more on the atmospheric retrievals, which means there are more simplifications and approximations for the land parameters, such as the fixed infrared (IR) emissivities in the identification of the Clear FOV, the homogenous surface in each FOV cluster for the CC algorithm, and the prior constraints on both Microwave (MW) and IR surface emissivities for the physical retrievals [20]. Consequently, the accuracies of the retrieved land surface parameters will be degraded if those underlying assumptions are violated. In addition, because a channel selection for CrIS data is adopted to speed up the retrieval process without losing obvious accuracies, the 


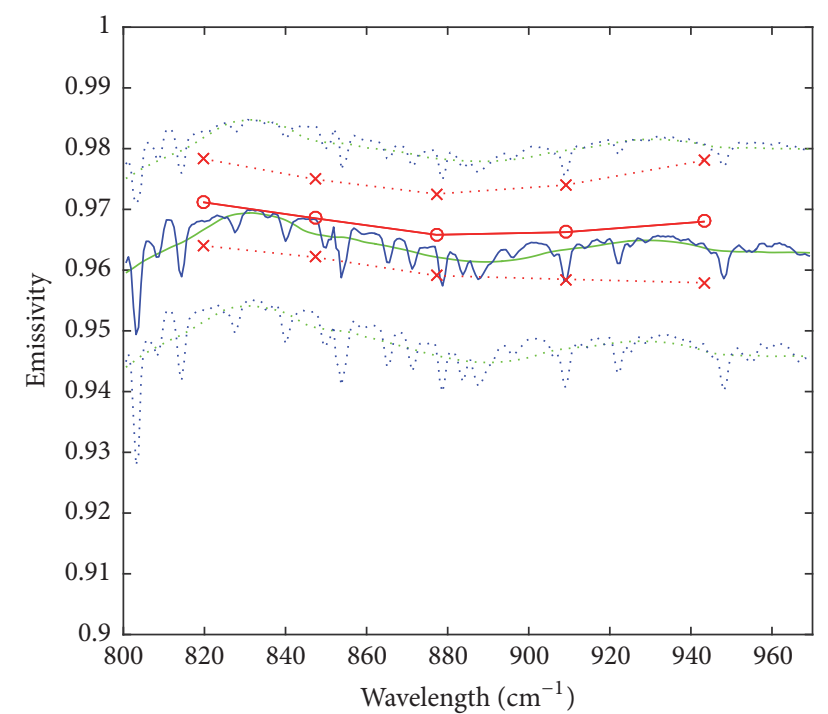

FIGURE 5: An overview of the LSE retrievals in May with the ARIS LSE product. The solid red line indicates the mean ARIS LSEs, and the dotted red lines show the standard deviation of the mean LSEs. Similarly, the blue lines are the mean and standard deviation of the original CrIS LSEs, while the green ones are for the smoothed CrIS LSEs.

retrieved LSEs are discrete. For example, just 12 IR LSEs, 12 IR surface reflectivities, and 1 LST, which are even not produced for users, are involved in the CrIS retrievals [20].

Unlike the complicated simultaneous approach used to retrieve atmospheric profiles, LST, and LSEs, in this paper, a simpler method with two steps are used, (1) atmospheric correction and (2) TES, to retrieve just the LST and LSEs from the CrIS data. By correcting for the atmospheric effects using the atmospheric reanalysis products and the RTTOV, the atmospherically corrected CrIS measurements at the surface level within each of the FOVs are first obtained. On this basis, the LST and LSEs can be then retrieved with the ISSTES algorithm with a finer spatial resolution approximately $14 \mathrm{~km}$ at nadir.

Compared with the present approach, our method is more effective and provides comparable accuracies for both the LST and LSEs with a higher spatial resolution. In addition, as no assumptions except a smooth LSE spectrum, which is easy to be satisfied in the range of 800-970 for most typical materials, is necessary for ISSTES algorithm, the retrieved LST and LSEs are more reasonable [12]. Furthermore, by smoothing the spectrum through mathematical methods, the retrieved LSEs are now given as a smoothed and continuous spectrum instead of a few channel LSEs, which is quite meaningful for various studies, such as object identification, resource exploration, process modeling, and weather forecasting $[1,2]$. There is a reason to believe that, with the development of hyperspectral instruments, using hyperspectral data to retrieve the LST and LSEs will be increasingly more accurate and inevitable to promote the development of relevant studies.

\section{Conclusions}

As a subsequent instrument of AIRS, the CrIS will continue to provide accurate atmospheric profiles for enhancing weather forecasting models and will facilitate both short- and longterm weather forecasting. However, compared with studies on the atmosphere, little attention has been paid to the retrievals of surface parameters using CrIS data, which are also critical for determining accurate climate variables.

In this paper, to simultaneously retrieve a continuous LSE spectrum and the LST from the CrIS data with the ISSTES algorithm, the NCEP atmospheric reanalysis data were used to provide the information of necessary atmospheric profiles for driving RTTOV. In addition, because of the difficulty in acquiring adequate ground measurements at large scale for validation, a cross-validation is used in this paper with the VIIRS LST and the AIRS emissivity products.

The results show that the overall RMSD of LSTs can be less than $1.4 \mathrm{~K}$. Considering the seasonal characters of atmospheric profiles, the RMSDs in May and August are larger than that of February and November, which may be caused by different amounts of water vapor in different seasons. When evaluating the retrievals of LSEs, because CrIS and AIRS have notably different spatial and temporal features, only the AIRS pixels covered with sufficient CrIS pixels within 6 hours of acquisition time are used in our study. The results show that the shapes of the retrieved LSE spectra are consistent with the AIRS product, and the overall RMSD is approximately $1.48 \%$, which is consistent with the RMSD of $1.4 \mathrm{~K}$ on LSTs. Although some uncertainty remains in the atmospheric correction process and CrIS data, the relevant retrievals in our study can provide comparable accuracy with current multispectral products. More importantly, the corresponding LSEs in our paper are provided as a continuum spectrum and contain much more spectral information than all current LSE products.

\section{Conflicts of Interest}

The authors declare that there are no conflicts of interest regarding the publication of this paper.

\section{Acknowledgments}

This study was supported by National Key Basic Research Program of China (973 Program, 2013CB733402); National Natural Science Foundation of China (41331171, 41571352, 41471297, and 41401394); Innovation Project of LREIS (O88RA801YA).

\section{References}

[1] Z.-L. Li, H. Wu, N. Wang et al., "Land surface emissivity retrieval from satellite data," International Journal of Remote Sensing, vol. 34, no. 9-10, pp. 3084-3127, 2013.

[2] Z.-L. Li, B.-H. Tang, H. Wu et al., "Satellite-derived land surface temperature: current status and perspectives," Remote Sensing of Environment, vol. 131, pp. 14-37, 2013. 
[3] J. A. Sobrino, J. C. Jiménez-Muñoz, G. Sòria et al., "Land surface emissivity retrieval from different VNIR and TIR sensors," IEEE Transactions on Geoscience and Remote Sensing, vol. 46, no. 2, pp. 316-327, 2008.

[4] Z. Wan and J. Dozier, "A generalized split-window algorithm for retrieving land-surface temperature from space," IEEE Transactions on Geoscience and Remote Sensing, vol. 34, no. 4, pp. 892-905, 1996.

[5] Z. Wan, "A physics-based algorithm for retrieving land-surface emissivity and temperature from eos/modis data," IEEE Transactions on Geoscience and Remote Sensing, vol. 35, no. 4, pp. 980-996, 1997.

[6] A. Gillespie, S. Rokugawa, T. Matsunaga, J. S. Cothern, S. Hook, and A. B. Kahle, "A temperature and emissivity separation algorithm for advanced spaceborne thermal emission and reflection radiometer (ASTER) images," IEEE Transactions on Geoscience and Remote Sensing, vol. 36, no. 4, pp. 1113-1126, 1998.

[7] A. R. Gillespie, M. S. Scholl, B. F. Andresen, T. Matsunaga, S. Rokugawa, and S. J. Hook, "Temperature and emissivity separation from Advanced Spaceborne Thermal Emission and Reflection Radiometer (ASTER) images," in Proceedings of the SPIE's 1996 International Symposium on Optical Science, Engineering, and Instrumentation, p. 82, Denver, CO, United States.

[8] Z. Wan, MODIS land-surface temperature algorithm theoretical basis document (LST ATBD), Institute for Computational Earth System Scienc, Santa Barbara, Calif, USA, 1999.

[9] Z. Wan and Z.-L. Li, "Radiance-based validation of the V5 MODIS land-surface temperature product," International Journal of Remote Sensing, vol. 29, no. 17-18, pp. 5373-5395, 2008.

[10] Joint Polar Satellite System (JPSS) VIIRS Land Surface Temperature Algorithm Theoretical Basis Document (ATBD), http://npp .gsfc.nasa.gov/sciencedocs/2015-06/474-00051_ATBD-VIIRSLST_A.pdf.

[11] B.-H. Tang, H. Wu, C. Li, and Z.-L. Li, "Estimation of broadband surface emissivity from narrowband emissivities," Optics Express, vol. 19, no. 1, pp. 185-192, 2011.

[12] C. C. Borel, "Surface emissivity and temperature retrieval for a hyperspectral sensor," in Proceedings of IEEE Conference on Geoscience and Remote Sensing, pp. 504-509, IEEE, July 1998.

[13] N. Wang, H. Wu, F. Nerry, C. Li, and Z.-L. Li, “Temperature and emissivity retrievals from hyperspectral thermal infrared data using linear spectral emissivity constraint," IEEE Transactions on Geoscience and Remote Sensing, vol. 49, no. 4, pp. 1291-1303, 2011.

[14] H. Wu, N. Wang, L. Ni, B.-H. Tang, and Z.-L. Li, "Practical retrieval of land surface emissivity spectra in $8-14 \mu \mathrm{m}$ from hyperspectral thermal infrared data," Optics Express, vol. 20, no. 22, pp. 24761-24768, 2012.

[15] H. H. Aumann, M. T. Chahine, C. Gautier et al., "AIRS/AMSU/HSB on the aqua mission: design, science objectives, data products, and processing systems," IEEE Transactions on Geoscience and Remote Sensing, vol. 41, no. 2, pp. 253-264, 2003.

[16] G. Chalon, F. Cayla, and D. Diebel, "IASI-An advanced sounder for operational meteorology," in Proceedings of the IAF, International Astronautical Congress, 52nd, Toulouse, France, 2001.

[17] D. Simeoni, M. Strojnik, P. Astruc et al., "Design and development of IASI instrument," in Proceedings of the Optical Science and Technology, the SPIE 49th Annual Meeting, p. 208, Denver, CO, USA.
[18] H. J. Bloom, “The cross-track infrared sounder (CrIS): a sensor for operational meteorological remote sensing," in Proceedings of the 2001 International Geoscience and Remote Sensing Symposium (IGARRS 2001), pp. 1341-1343, July 2001.

[19] X. Liu, S. Kizer, C. Barnet et al., "Retrieving atmospheric temperature and moisture profiles from SUOMI NPP CrIS/ATMS sensors using CrIMSS EDR algorithm," in Proceedings of the 2012 32nd IEEE International Geoscience and Remote Sensing Symposium, IGARSS 2012, pp. 1956-1959, Munich, Germany, July 2012.

[20] Joint Polar Satellite System (JPSS) Algorithm Theoretical Basis Document For the Cross Track Infrared Sounder (CrIS) Volume II, Environmental Data Records (EDR), http://npp.gsfc .nasa.gov/sciencedocs/2015-06/474-00056_ATBD-CrIS-EDR_B .pdf.

[21] E. Bartholomé and A. S. Belward, "GLC2000: a new approach to global land cover mapping from earth observation data," International Journal of Remote Sensing, vol. 26, no. 9, pp. 19591977, 2005.

[22] Joint Polar Satellite System (JPSS) Cross Track Infrared Sounder (CrIS) Sensor Data Records (SDR) Algorithm Theoretical Basis Document (ATBD), http://npp.gsfc.nasa.gov/sciencedocs/201506/474-00032_ATBD-CrIS-SDRs_C.pdf.

[23] Y. Han, H. Revercomb, M. Cromp et al., "Suomi NPP CrIS measurements, sensor data record algorithm, calibration and validation activities, and record data quality," Journal of Geophysical Research Atmospheres, vol. 118, no. 22, pp. 12734-12748, 2013.

[24] S. Kizer, X. Liu, A. Larar et al., "Porting and testing NPOESS crimss EDR algorithms," in Proceedings of the 2010 30th IEEE International Geoscience and Remote Sensing Symposium, IGARSS 2010, pp. 1067-1069, Honolulu, Hawaii, USA, July 2010.

[25] E. Kalnay, M. Kanamitsu, R. Kistler et al., "The NCEP/NCAR 40-year reanalysis project," Bulletin of the American Meteorological Society, vol. 77, no. 3, pp. 437-471, 1996.

[26] C. Cao, X. Xiong, R. Wolfe et al., "Visible Infrared Imaging Radiometer Suite (VIIRS) Sensor Data Record (SDR) Users Guide, NOAA," Tech. Rep., College, Park, MD, USA, 2013.

[27] P. C. Guillevic, J. C. Biard, G. C. Hulley et al., "Validation of Land Surface Temperature products derived from the Visible Infrared Imaging Radiometer Suite (VIIRS) using groundbased and heritage satellite measurements," Remote Sensing of Environment, vol. 154, pp. 19-37, 2014.

[28] H. Li, D. Sun, Y. Yu et al., "Evaluation of the VIIRS and MODIS LST products in an arid area of Northwest China," Remote Sensing of Environment, vol. 142, pp. 111-121, 2014.

[29] M. Gunson, M. Chahine, and H. Aumann, "AIRS-Team Retrieval For Core Products and Geophysical Parameters-Level 2," https://disc.gsfc.nasa.gov/AIRS/documentation/v6_docs/ v6releasedocs-1/V6_L2_Product_User_Guide.pdf.

[30] G. C. Hulley, S. J. Hook, E. Manning, S. Lee, and E. Fetzer, "Validation of the Atmospheric Infrared Sounder (AIRS) version 5 land surface emissivity product over the Namib and Kalahari deserts," Journal of Geophysical Research, vol. 114, no. D19, 2009.

[31] J. Hocking, P. J. Rayer, D. Rundle, R. W. Saunders et al., RTTOV v11 Users Guide, NWP-SAF report, Met. Office, Exeter, UK, 2013.

[32] C. Borel, "Error analysis for a temperature and emissivity retrieval algorithm for hyperspectral imaging data," International Journal of Remote Sensing, vol. 29, no. 17-18, pp. 50295045, 2008.

[33] P. M. Ingram and A. H. Muse, "Sensitivity of iterative spectrally smooth temperature/emissivity separation to algorithmic 
assumptions and measurement noise," IEEE Transactions on Geoscience and Remote Sensing, vol. 39, no. 10, pp. 2158-2167, 2001.

[34] H. Li, Q. Liu, Y. Du, J. Jiang, and H. Wang, "Evaluation of the NCEP and MODIS atmospheric products for single channel land surface temperature retrieval with ground measurements: a case study of HJ-1B IRS data," IEEE Journal of Selected Topics in Applied Earth Observations and Remote Sensing, vol. 6, no. 3, pp. 1399-1408, 2013.

[35] H. Wu and Z.-L. Li, "Scale issues in remote sensing: A review on analysis, processing and modeling," Sensors, vol. 9, no. 3, pp. 1768-1793, 2009.

[36] H. Wu, B.-H. Tang, and Z.-L. Li, "Impact of nonlinearity and discontinuity on the spatial scaling effects of the leaf area index retrieved from remotely sensed data," International Journal of Remote Sensing, vol. 34, no. 9-10, pp. 3503-3519, 2013. 

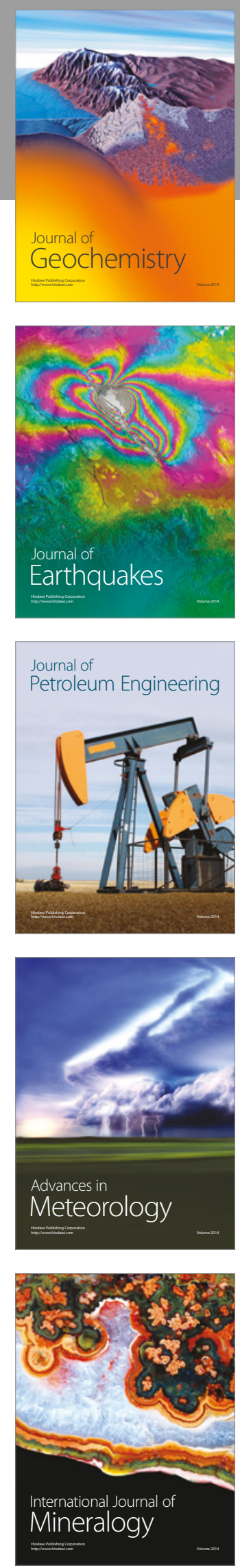
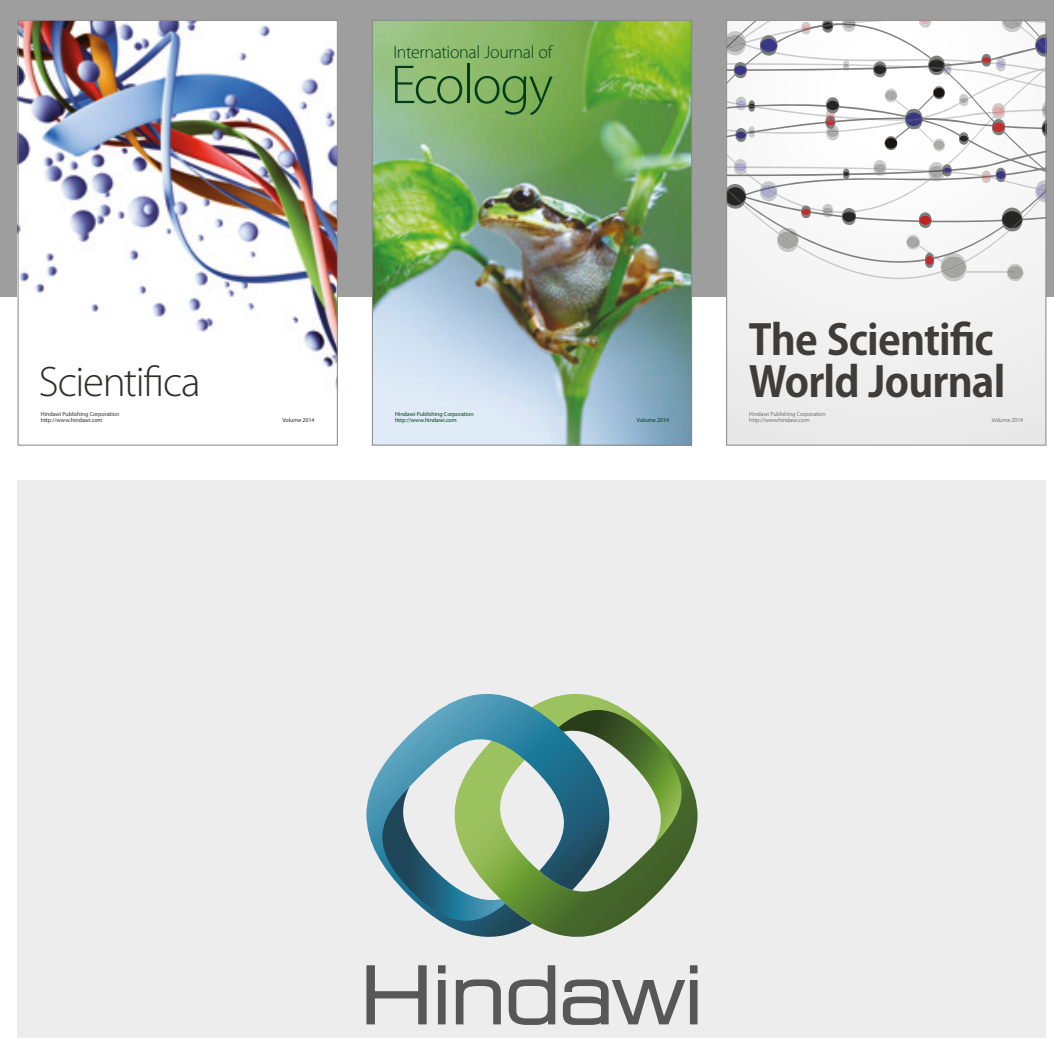

Submit your manuscripts at

https://www.hindawi.com
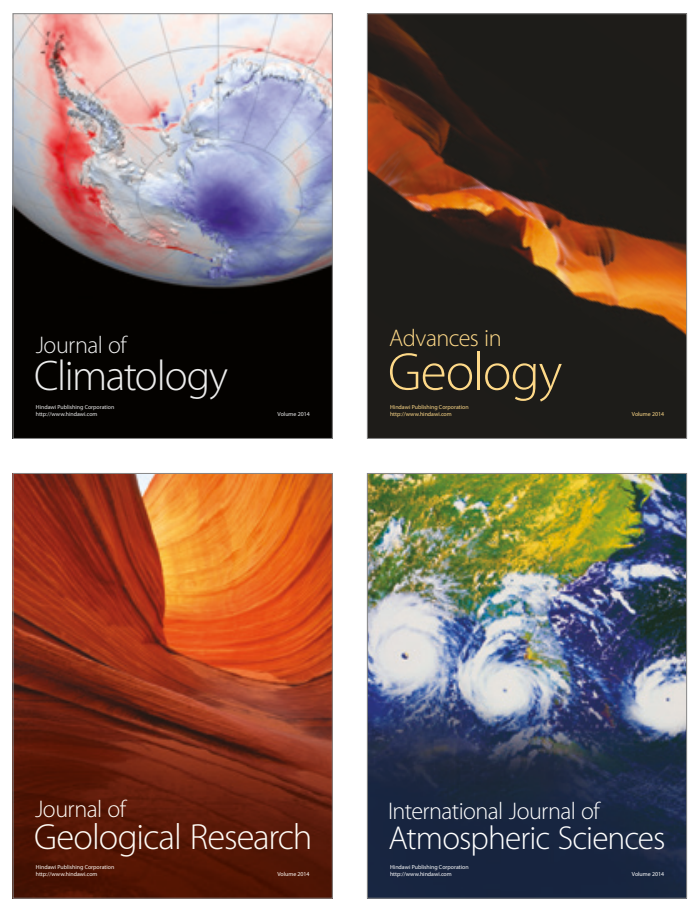

The Scientific

World Journal
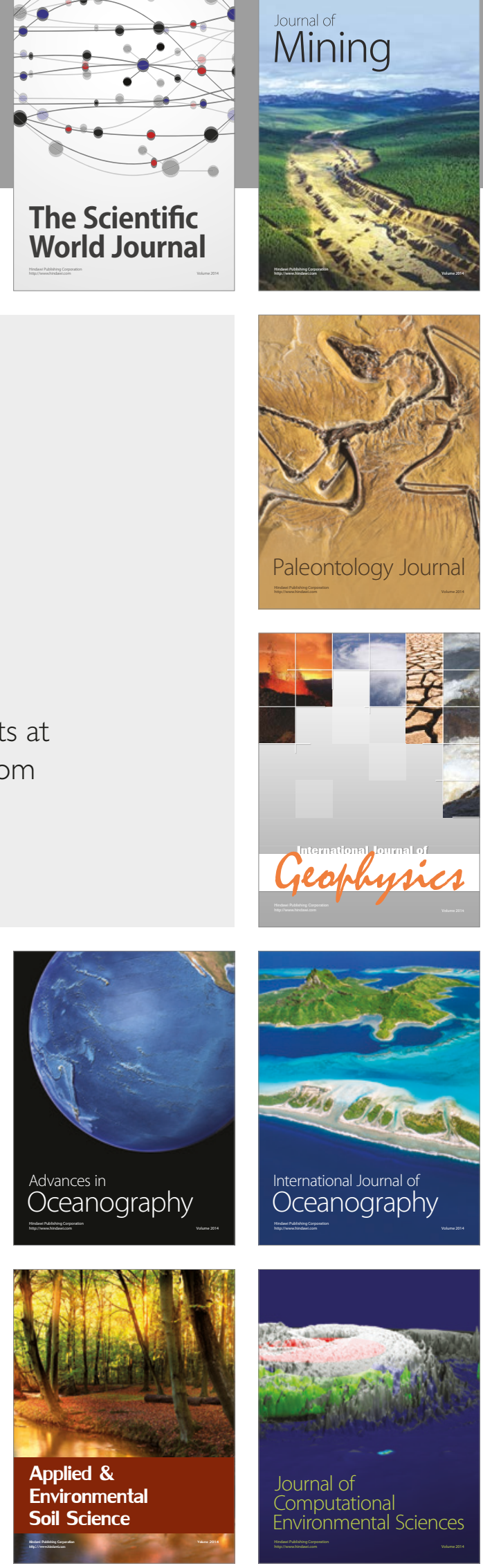